\title{
The SIR Epidemiology Model in Predicting Herd Immunity
}

Joanna Nicho

University of South Florida

Advisors:

Arcadii Grinshpan, Mathematics and Statistics

Gordon Fox, Integrative Biology

Problem Suggested By: Gordon Fox

Follow this and additional works at: https://digitalcommons.usf.edu/ujmm

Part of the Mathematics Commons

UJMM is an open access journal, free to authors and readers, and relies on your support:

Donate Now

\section{Recommended Citation}

Nicho, Joanna (2010) "The SIR Epidemiology Model in Predicting Herd Immunity," Undergraduate Journal of Mathematical Modeling: One + Two: Vol. 2: Iss. 2, Article 8.

DOI: http://dx.doi.org/10.5038/2326-3652.2.2.8

Available at: https://digitalcommons.usf.edu/ujmm/vol2/iss2/8 


\title{
The SIR Epidemiology Model in Predicting Herd Immunity
}

\begin{abstract}
The Simple Epidemic Model uses three states to describe the spread of an infection: the susceptible (S), the infected (I), and the recovered (R). This model follows the trend of an infection over time and can predict whether an infection will spread. Using this model, epidemiologists may calculate the percentage of the population that needs to be vaccinated in order to provide a population immunity from a disease. This study will compare the vaccination percentage required for herd immunity to measles, mumps, and rubella against the current percentage of vaccinated individuals.
\end{abstract}

\section{Keywords}

Simple Epidemic Model, Vaccination, Herd Immunty

\section{Creative Commons License}

(c) (i) ()

This work is licensed under a Creative Commons Attribution-Noncommercial-Share Alike 4.0 License. 


\section{TABLE OF CONTENTS}

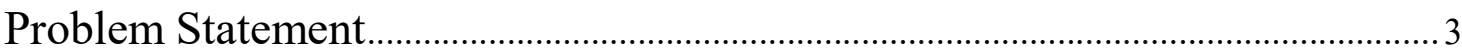

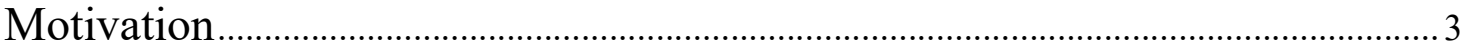

Mathematical Description and Solution Approach ................................................ 4

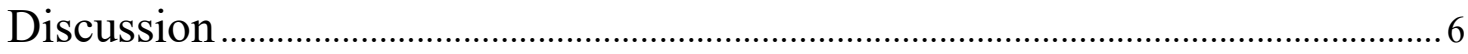

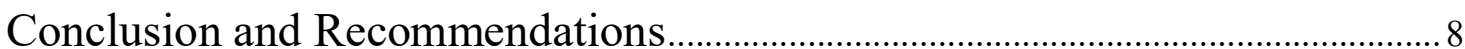

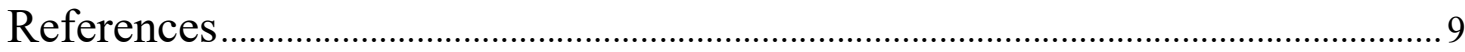

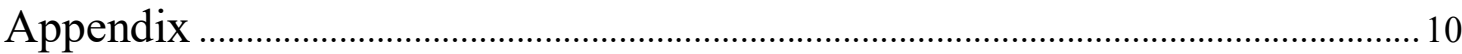




\section{PROBLEM STATEMENT}

In this study, we will calculate the percentage of people that need to be vaccinated for herd immunity for measles, mumps, and rubella (MMR). After this number is calculated, it will be compared to the percentage of unvaccinated children. Some parents choose not to vaccinate their children based on beliefs that the MMR vaccine increases the chances of autism. If parents reject vaccination for their children, will this have an overall effect on herd immunity? If so, what can be done to avoid this?

\section{MOTIVATION}

In 1998, the British medical journal Lancet published an article (now retracted) implying a link between the Measles, Mumps, and Rubella vaccine and autism (Wakefield et al. 1998). The article stated that there was no clear connection between the MMR vaccine and autism. It concluded that, although more research was needed to prove or disprove the link, there were possible environmental causes for the onset of the disease. This conclusion implicated the vaccine as one of the possible environmental causes.

With this new information, parents that would have otherwise given their children the MMR vaccine began to question whether they should allow their children to be vaccinated. Since 1998, an increasing number of parents have chosen not to vaccinate their children.

A recent study that assessed parental concerns about vaccines found that over $90 \%$ of parents believe that vaccines are a good way to keep their children healthy (Freed et al, 2009). However, nearly $12 \%$ of parents have refused at least one vaccination for their children (Freed et 
al, 2009). Furthermore, $25 \%$ of parents strongly believe that "some vaccines cause autism" (Freed et al, 2009).

While many parents continue to immunize their children, the number of parents that believing in a link to autism is still very high. If parents decide not to vaccinate their children, the prevalence of measles, mumps and rubella (which have been dramatically decreased around the world due to vaccinations) will begin to rise again. Stopping the spread of MMR requires the vaccination of most children.

The percentage of people that need to be vaccinated can be calculated using the SIR model. This information is used by public health officials to determine how many people should be vaccinated so that the entire community will be immune (herd immunity). If enough people are immune, a disease will not spread. The SIR model is a valuable tool in public health because it suggests the requirements to prevent infections. Through vaccinations and the concept of herd immunity, disease can potentially be eradicated. The eradication of small pox was achieved through such vaccination.

\section{MATHEMATICAL DESCRIPTION AND SOLUTION APPROACH}

The simple epidemic model (SIR model) was used to determine the number of people that need to be vaccinated to create herd immunity. It was also used to determine whether the statistics on people hesitating or refusing to receive vaccines may cause a detrimental effect on the goal of herd immunity. 
The SIR model divides the population into three classes: $S$ for the susceptible, $I$ for the infected, and $R$ for the recovered or removed. This is illustrated in Figure 1 in the appendix. Immunization moves individuals from the $S$ section to the $R$ section. The model is based on several assumptions (Stroyan, 2009). The first assumption is that all individuals of the population must fit into one of the categories $(S, I$, or $R)$. The second assumption is that the population is large and relatively fixed. This basic model will assume that the births and deaths in the population are similar enough that they do not need to be included in the equation. The last assumption is that the population is well mixed so that every individual has contact with the same number of people.

The dynamics of the model are described by the following equations (Neuhauser, 2004):

$$
\begin{aligned}
& \frac{d S}{d t}=-b S I \\
& \frac{d I}{d t}=b S I-a I \\
& \frac{d R}{d t}=a I .
\end{aligned}
$$

Here, $a$ and $b$ are positive constants (basic epidemiology models) (Hethcote, 1989). The infection rate, $b$, is the average rate that an infected individual infects a susceptible person. The recovery rate, $a$, is found by dividing 1 by the number of days that the infection can spread. Using the above equations, we see:

$$
\frac{d S}{d t}+\frac{d I}{d t}+\frac{d R}{d t}=-b S I+b S I-a I+a I=0
$$

Integrating with respect to $t$, we find: 


$$
S(t)+I(t)+R(t)=N
$$

where $N$ denotes the population size.

The concept of herd immunity uses the basic reproductive rate denoted by $R_{0}$. This value predicts whether a disease can spread. The equation is:

$$
R_{0}=\frac{b}{a} S(0)
$$

If the value is less than 1 , then the disease will not spread. Since we are discussing communicable disease, we consider cases such that $R_{0}>0$. The higher the value $R_{0}$, the more infectious the disease will be. The values of $R_{0}$ for measles, mumps, and rubella are 16,12 , and 7 respectively (Hethcote, 2000).

Finally, herd immunity depends on the contact number of the disease, denoted $c$. The contact number is defined such that $R>1-\frac{1}{c}$ (Hethcote, 1989). Herd immunity is achieved when $c(a-R)<1$ (Hethcote, 2000).

\section{DISCUSSION}

The percentages of vaccinations required for herd immunity were based on the $\mathrm{R}_{0}$ and contact numbers provided by a previous study. Measles, with a $R_{0}$ value of 16 and a contact number of 12.3 , requires $92 \%$ of the population to be vaccinated to achieve herd immunity. The vaccination percentage is high due to the infectious nature of measles which is reflected by its high $R_{0}$ value. Mumps with a $R_{0}$ value of 12 and a contact number of 8.1 requires $88 \%$ of the population to be vaccinated for herd immunity. Rubella with a $R_{0}$ of 7 and a contact number of 7 
requires $86 \%$ of the population to be vaccinated. Typically the inoculation for measles, mumps and rubella are administered at the same time with a MMR vaccine. The vaccine must be given to at least $92 \%$ of the population for the herd immunity to be achieved. In fact, according to the World Health Organization, $92 \%$ of Americans are vaccinated with the MMR vaccine (2009). This number should theoretically ensure herd immunity.

As the number of vaccinations increases, the number of people in the $R$ state also increases. As was explained earlier, $S+I+R=N$ where $N$ is the total population. Therefore, $S$ is decreasing while $R$ is increasing. In the graphs seen in Figure 2 of the Appendix, $S$ is seen decreasing over time while $R$ is increasing. The number of those susceptible, $S$, is being "used up" by shifting to either the vaccinated or non-vaccinated individuals.

According to the survey on vaccines and autism, 25\% of parents have hesitations about the vaccine. If all of these parents were to stop vaccinating their children, only $75 \%$ of the population would be vaccinated. This is significantly lower than the amount of vaccinations required for herd immunity and could lead to a potential outbreak of all three diseases. The implications of these calculations indicate the importance of educating the population on vaccinations. Since these infectious diseases are matter of public policy, schools now require proof of immunizations for enrollment. 


\section{CONCLUSION AND RECOMMENDATIONS}

The results of this study showed that a high percentage of the population must be vaccinated in order to maintain herd immunity due to the highly contagious nature diseases such as measles, mumps and rubella. These diseases are preventable through vaccination; moreover it is practical to vaccinate large portions of the population. The public health officials need to continue to actively encourage parents to vaccinate their children. Also, parents need to realize that vaccinations are only effective if a large portion of the population receives the vaccination.

Future studies may explore the effects of vaccination over a greater period of time and incorporate sampled birth and death statistics. These additions will provide a more accurate, long-term description. 


\section{REFERENCES}

Bedford, H.E. and Elleman, D.A. "MMR Vaccine and Autism." BMJ. 6 Feb 2010- v 340

Freed, G.L. et al. "Parental Vaccine Safety Concerns in 2009.” Pediatrics. 1 Mar 2010.

Keeling, Matt "The Mathematics of Diseases" 2001.

$<$ http://plus.maths.org.uk/issue14/features/diseases/index-gifd.html $>$.

Hethcote, Herbert. "The Mathematics of Infectious Diseases." SIAM Review. 2000: v 42.4.

Hetbcote, Herbert. "Three Basic Epidemiological Models.” Biomathematics. 1989: v 18.

Ng et al. "Typical Dynamics for the SIR Model.” BMC Infectious Diseases. 2003: v 3.19

Stroyan, Keith. "Using Calculus to Model Epidemics."

$<$ http://www.math.uiowa.edu/ stroyan/CTLC3rdEd/3rdCTLCText/Chapters/Ch2.pdf $>$.

Wakefield, AJ et al. "Ileal-lymphoid-nodular hyperplasia, non-specific colitis, and pervasive developmental disorder in children." The Lancet. 1998: v 351, 9103 pp.637-641

"Immunization Profile-United States of America." WHO. 2009

$<\mathrm{http}: / /$ www.who.int/immunization_monitoring/en/globalsummary/countryprofileresult.cfm>. 


\section{APPENDIX}

Figure 1:

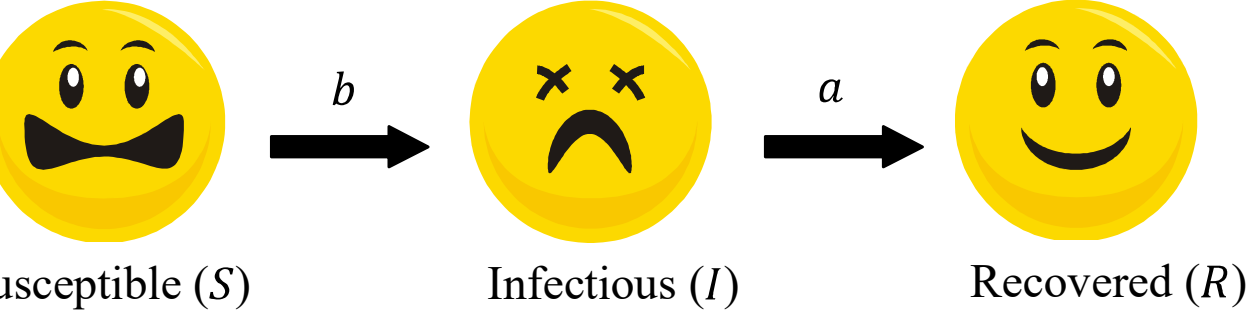

This figure illustrates the SIR model flow from one state to another.

Figure 2:

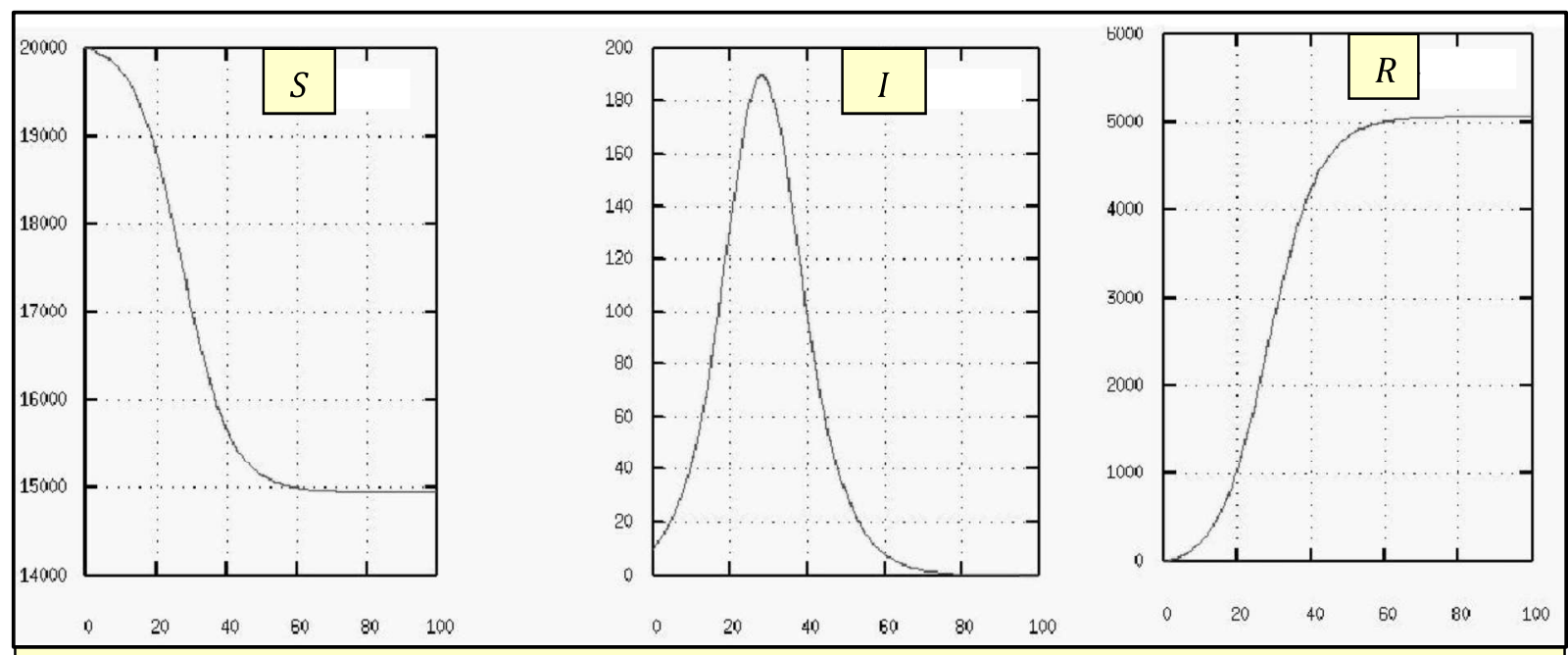

This figure ( $\mathrm{Ng}$ et al, 2003) illustrates the typical trends seen in the SIR model:

- $S$ decreases until there are very little susceptible left

- I peaks but then decreases over time

- $R$ increases as the vaccinations are given and people recover from the infection 